\title{
Conversion Energy from the Movement of the Solar System Through Universal Pressure: Reflections in Seismic Events and Global Temperatures
}

\author{
Michael A. Persinger \\ Laurentian University, Sudbury, Ontario P3E 2C6, Canada \\ E-mail address: mpersinger@laurentian.ca
}

\begin{abstract}
The velocity $\left(\sim 242 \mathrm{~km} \cdot \mathrm{s}^{-1}\right)$ of the Solar System around the galactic center within the universal pressure $\left(\sim 10^{-10}\right.$ Pascal $)$ produces energies within the earth's volume that is equivalent to that released by the sum of all earthquakes per unit time. The available energy within the earth and solar volume from the expected spatial variations of this pressure along this perimeter, which requires about 250 million years to traverse, can accommodate the increased geomagnetic activity from the expanding solar corona over the last approximately 100 years as well as the increase in global warming. Inferences of a varying structure of space that may explain the periodicity and range in solar cycles as well as anomalous minimums (such as the Maunder phenomenon) suggest a central galactic singularity with spatial ripples exhibiting peak-to-peak troughs that approximate the earth's circumference and frequencies in the order of 7 to $8 \mathrm{~Hz}$. The precise velocity-universal pressure flux density may also explain the millilux-range magnitude of the earth's night (air) glow. These results and the application of these concepts indicate that origins of seismicity, slow drifts in the intensity of geomagnetic activity, and global warming (and cooling) trends are products of differential interactions with quantitative fluctuations in sub-matter space and that the subtle variations encountered as the Solar System moves along this $10^{21} \mathrm{~m}$ perimeter may be more significant than previously assumed.
\end{abstract}

Keywords: Solar System; Seismicity; Geomagnetic Activity; Global Warming; Night Glow; Electron Mass Equivalence

\section{INTRODUCTION}

The orbital movements of earth around a G2 star and the correlative seasons may reflect the representation of closed and redundant processes. However this circularity does not occur in the same absolute space. The Solar System moves through space around the galactic center at approximately $2.42 \cdot 10^{5} \mathrm{~m} \cdot \mathrm{s}^{-1}$. Given the Sun's distance of $\sim 2.77 \cdot 10^{20} \mathrm{~m}$ from the center and assuming an orbit with minimal eccentricity, the time required for one galactic orbit would be about 250 million years [1].

The nature of the spatial structure below the levels of discourse that define matter $\left(\sim 10^{-}\right.$ $15 \mathrm{~m})$ and between this organization of space and Planck's length $\left(\sim 10^{-35} \mathrm{~m}\right)$ is unclear. Recently calculations suggest that a significant component of the energy within the universe is contained within the geometry of sub-matter space [2]. The concept is compatible with Borowski's model [3] that motions of planets around the sun and movements of galaxies are 
by means of differential pressures from dark matter. According to Borowski, "the whole planetary system..moves in a solvent which is dark matter".

If such "dark matter" or its functional equivalent reflects an intrinsic energy coupled to the total life time ("final epoch") of the universe [4], then its quantity and variations encountered continuously during the Solar System's galactic orbit could affect fundamental phenomena on earth. Here I exam the potential contribution of the energy within the hypothetical organization of space that is traversed by the orbit of the solar system around the galactic center. Quantitative convergence of energies available from this movement through a universal pressure to global seismicity and fluctuations in global temperature are discussed.

\section{UNIVERSAL PRESSURE AND EARTHQUAKE ENERGIES}

Dimensional analysis for pressure indicates it is the product density $(\rho)$ and the square of velocity. Assuming $1.67 \cdot 10^{-27} \mathrm{~kg} \cdot \mathrm{m}^{-3}$ for the average universal density and the square of the velocity of light $\left(3 \cdot 10^{8} \mathrm{~m} \cdot \mathrm{s}^{-1}\right)$ in a vacuum, an intrinsic dynamic pressure of $1.5 \cdot 10^{-10} \mathrm{~Pa}$ $\left(\mathrm{kg} \cdot \mathrm{m}^{-1} \cdot \mathrm{s}^{-2}\right)$ would be present [5]. That this is related to a universal constant, Newtonian $\mathrm{G}$ $\left(6.67 \cdot 10^{-11} \mathrm{~kg}^{3} \cdot \mathrm{m}^{-1} \cdot \mathrm{s}^{-2}\right)$ can be demonstrated by the tensor, relating both, which is $\mathrm{m}^{4} \mathrm{~kg}^{-2}$.

For the two values, universal pressure and $G$, to be equivalent and assuming the four dimensional space of $10^{104} \mathrm{~m}^{4}$ (the quadruple of $10^{26} \mathrm{~m}$ ), the mass must be $10^{104} \mathrm{~kg}^{2}$ to achieve an identity. Hence the mass of the universe would be $\sim 10^{52} \mathrm{~kg}$ ( or $\sim 10^{69} \mathrm{~J}$ ) which is within an order of magnitude of solutions from several other approaches. This convergence suggests that the existence of an intrinsic dynamic pressure, of about $1.5 \cdot 10^{-10} \mathrm{~Pa}$, averaged across the universe may be a significant entity.

Energy is the product of pressure and volume. If the earth as a component of the solar system is moving through this universal pressure there would be a discrete amount of energy available within specific volumes. The median depth for the total numbers of global earthquakes for different integer magnitudes for the years 2009 through 2013 was a depth of $\sim 60 \mathrm{~km}$ according to data from the USGS. The volume of this shell is $3.03 \cdot 10^{19} \mathrm{~m}^{3}$.

The energy available from the universal pressure as the Solar System moves around the galaxy within this shell would be $4.5 \cdot 10^{9} \mathrm{~J}$ per unit time. In this instance $1 \mathrm{~s}$ is assumed for convenience. This is the energy equivalent of an approximately $3 \mathrm{M}$ seismic event. Consequently the order of magnitude of energy available from the universal pressure would be sufficient to add to intrinsic seismic energy that is discerned as earthquakes.

Vares and Persinger [6] suggested that the relative paucity in the distribution of earthquakes within the $\mathrm{M}=3.5$ to 4.0 range, specifically in the trough between M 3.6 and 3.7, approached the energies associated with Planck's Length. The related time increment approached the cutoff frequency for the Zero Point Fluctuation force coupled to gravity [7]. They suggested a conduit might exist between intrinsic features of space-time and geophysical processes that could contribute to the transformation of virtual particles into real particles [8] or to the equilibrium of this process.

The associated energy for the threshold for such a conduit is $1.2 \cdot 10^{10} \mathrm{~J}$ and would be the magnitude induced from the universal pressure within a shell with a depth of about 150 to 160 $\mathrm{km}$. The United States Geological Survey (USGS) data indicate that the average depths of 3.5 $(\mathrm{n}=63,446)$ and $4.0(\mathrm{n}=59,773)$ events were $56.5 \mathrm{~km}(\mathrm{SD}=86.6)$ and $58.2(\mathrm{SD}=94.3)$, respectively. The energy available from the universal pressure within a shell $57 \mathrm{~km}$ thick from the surface would be sufficient to generate potentially one of these events every $\sim 2 \mathrm{~s}$. 
The more profound implication is when the total energy from $\mathrm{M}<8.1$ earthquakes is calculated. The cumulative energy $\left(4.5 \cdot 10^{9} \mathrm{~J}\right.$ per s) within the shell of the average depth of earthquakes over the 5 years of the data for total global earthquakes is $\sim 7.1 \cdot 10^{17} \mathrm{~J}$. The total numbers of $7.0 \mathrm{M}$ events during this time was 98 . Assuming the upper value of $2 \cdot 10^{15} \mathrm{~J}$ per M 7 event, the total energy would be $\sim 2 \cdot 10^{17} \mathrm{~J}$. In comparison the 139,265 events between M 2.00 and 2.5, each with a magnitude of $6.3 \cdot 10^{7} \mathrm{~J}$, would exert a cumulative energy of only $8.8 \cdot 10^{12} \mathrm{~J}$.

In other words, all $(n=518,730)$ of the energy associated with all of the earthquakes below 8.1 during that five year interval could be accommodated by the energy available within the volume of the shell defined by the average depth of earthquakes from the presence of the universal pressure as the earth moves through intragalactic space. If the depth is expanded to include deep earthquakes, then the available energy could accommodate every seismic event within the earth's crust.

\section{CHANGING INTERPLANETARY MAGNETIC FIELD INTENSITES AND GLOBAL TEMPERATURE FLUCTUATIONS}

According to El-Borie and Al-Thoyaib [9] the approximately $1.1^{\circ} \mathrm{C}$ increase in global mean temperature since about 1877 is unlikely to be totally due internal climate variability. Over the past century the approximately 0.4 to $0.8{ }^{\circ} \mathrm{C}$ increase in oceanic temperatures and 0.8 to $2.2^{\circ} \mathrm{C}$ increase in air temperatures within the lower troposphere have been strongly correlated with the solar cycle [10]. During this same period there has been an expansion of the solar corona and an increase in the average global geomagnetic activity. Between 1905 and 1965 this correlation was $r=0.55$ [11].

Considering the dynamic pressure from the average velocity of the solar wind (468 $\mathrm{km} \cdot \mathrm{s}^{-1}$ ) and a density of 10 protons per cc, the value is about 3.5 nanoPascal. Persinger [11] calculated that within a volume that extends one earth radius $(6,378 \mathrm{~km})$ into space the net increase in energy would be equivalent to an increased of $16 \mathrm{nT}$. This was within measurement error of the average increase in aa values reported by Lockwood et al [12]. The origin of the increase in temperature from within a more interplanetary source rather than human or other terrestrial sources was suggested by the comparable increase in inferred surface temperature on Mars [13].

Application of the concepts of the current approach suggests that the origin of the increased energy from persistent interaction with the universal pressure could contribute to the increase in global temperatures and geomagnetic activity. Employing the equation:

$$
\mathrm{B}^{2}=(\mathrm{J} \cdot 2 \mu) \cdot \mathrm{m}^{-3}
$$

where, $\mathrm{J}$ is the energy available within the earth's volume $\left(1.09 \cdot 10^{21} \mathrm{~m}^{3}\right)$ from the universal pressure $\left(1.5 \cdot 10^{-10} \mathrm{~kg} \cdot \mathrm{m}^{-1} \mathrm{~s}^{-2}\right)$ or $1.64 \cdot 10^{11} \mathrm{~J}$ and $\mu$ is magnetic permeability of a vacuum $\left(4 \pi \cdot 10^{-7} \mathrm{~N} \cdot \mathrm{A}^{-2}\right)$, the equivalent magnetic field strength is $\sim 19 \mathrm{nT}$. This is within the range of increase in the variation of the geomagnetic activity measured empirically.

If we assume the Central Limit Theorem operates within galactic spatial frames, then there should be a standard deviation for the variation around the central tendency. A conservative range for the coefficient of variation (SD or standard deviation divided by the mean) for a normal distribution is about 0.33 . This would mean that the actual universal pressure values would range from 0.5 to $2.5 \cdot 10^{-10}$ through $95 \%$ of the space and time. The 
equivalent range in geomagnetic activity from those intrinsic energies as calculated from (1) would range between $11 \mathrm{nT}$ and $25 \mathrm{nT}$. The average increase in global aa (average antipodal) geomagnetic values since 1900 has been about $16 \mathrm{nT}$ which is well within this range of this variation.

A similar solution occurs within the volume of the Sun $\left(1.13 \cdot 10^{28} \mathrm{~m}^{3}\right)$. The energy available from the universal pressure would be $1.68 \cdot 10^{18} \mathrm{~J}$. The magnetic field strength equivalent, according to equation (1) would be about $19 \mathrm{nT}$. Consequently the increase in geomagnetic activity associated with the expansion of the solar corona could be accommodated by the expected variations in quantity of the universal pressure parameter.

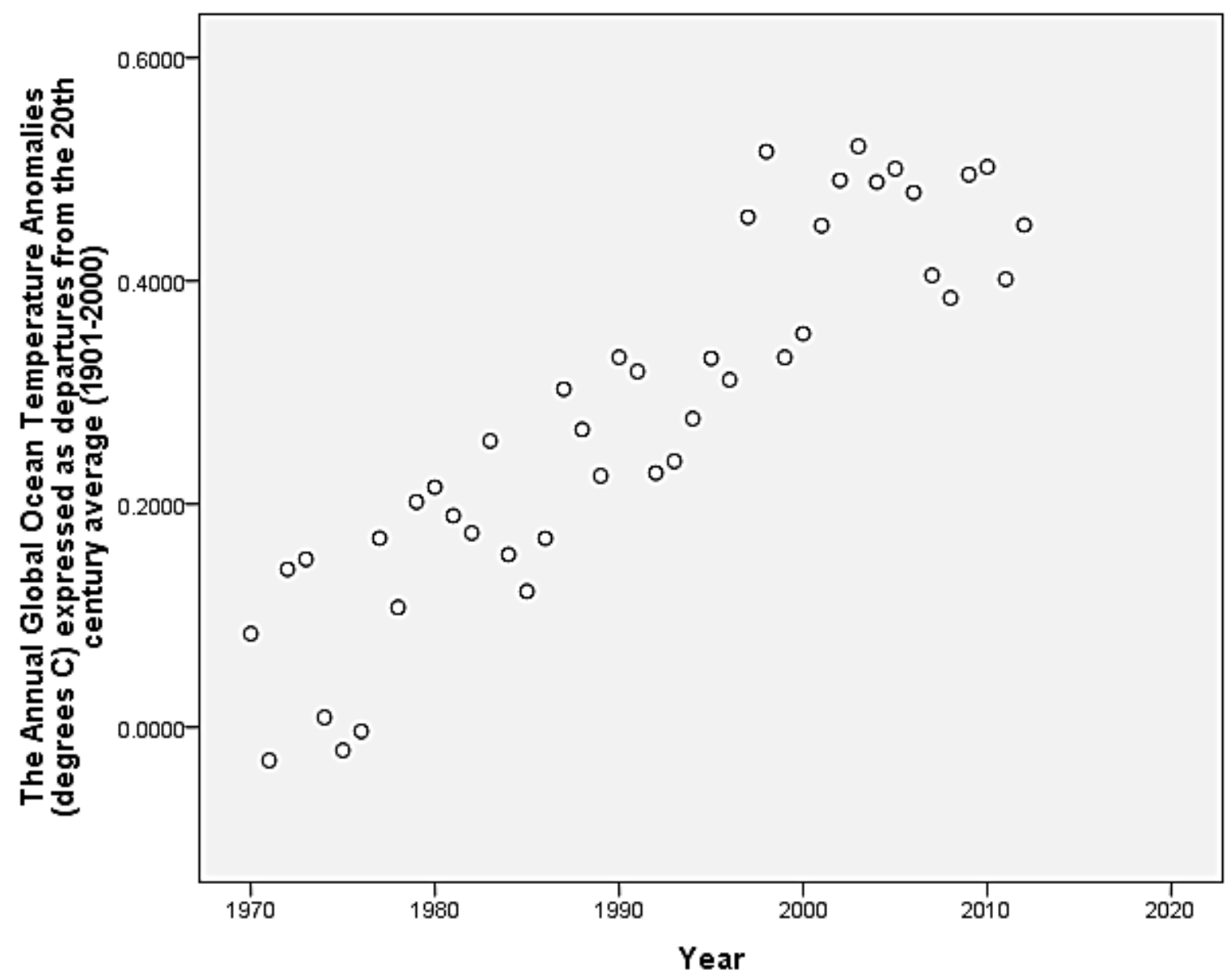

Figure 1. Scattergram of annual global ocean temperature anomalies between 1970 and 2013. Note the asymptote that began around the year 2005 .

The contribution of this additional energy to the oceans would result in an average volume increase by $\sim 0.5{ }^{\circ} \mathrm{C}$ within the time frame of the observations. The volume of the oceans is $\sim 1.3 \cdot 10^{18} \mathrm{~m}^{3}$ which means the intrinsic energy from the universal pressure $\left(1.5 \cdot 10^{-10}\right.$ $\mathrm{Pa}$ ) would be $1.95 \cdot 10^{8} \mathrm{~J}$ per s. Assuming the classic definition that a calorie of heat is required to change the temperature of $1 \mathrm{~g}$ of water by $1{ }^{\circ} \mathrm{C}$ [14], the amount of energy (as heat) to increase $1.3 \cdot 10^{24}$ cc of ocean $0.5{ }^{\circ} \mathrm{C}$ would be $\sim 0.65 \cdot 10^{24}$ erg or $\sim 0.66 \cdot 10^{17} \mathrm{~J}$. With this persistent addition of energy, assuming minimum loss, the time required to elevate the volume 
by that temperature would be $0.33 \cdot 10^{9} \mathrm{~s}$ or about 10.7 years; this is effectively one solar cycle.

However the gradual increase in global oceans temperatures would suggest that the value for the universal pressure remained elevated. Assuming a maintained increase of 1 standard deviation, or $0.5 \cdot 10^{-10} \mathrm{~Pa}$, the time required for this elevation in ocean temperature to occur would be about 32 years. A maintained elevation of 0.5 standard deviations would require 64 years to produce the current values.

The biosphere, often defined as the distance between the earth's surface and the height of $10 \mathrm{~km}$ (the troposphere), occupies a volume $\left(5.1 \cdot 10^{18} \mathrm{~m}^{3}\right)$ that is remarkably similar to that of the oceans. Even a conservative estimate of loss of the energy through convection [15] from the oceans into the atmosphere, would suggest that the current elevated drift would have required between 50 and 100 years. This duration is consistent with observation [9].

If the increase in global ocean temperatures were due to cumulative human activities, then one would expect the trend to continue indefinitely. However as seen in Figure 1, the increase stopped around the year 2005. The correlation (Pearson $r$ ) before 2000 was $r=0.85$ $(p<.001)$. On the other hand the correlation between time and global ocean temperatures during the 8 years since 2005 was $r=-0.22$ and was not significant statistically. This change would be consistent with the predicted diminished value of the universal pressure which would have contributed as well to the reduced solar activity during 2013 which has been the lowest in approximately 100 years. If the present concepts are applicable this means the Solar System is now encountering a diminished quantity for universal pressure.

\section{IS THERE AN INTRINSIC STRUCTURAL VARIATION IN GALACTIC SPACE?}

The origin or cause of the "solar cycle", whose mean duration is about 10.4 years has been attributed to complex interactions between the barycenter of Jupiter's orbit and the solar mass. The presence of the range in the duration ( 7 to about 17 years) of the solar cycle as well as the magnitude of the peaks and troughs of this activity, as inferred by numbers of sunspots or more recently discrete $\mathrm{GHz}$ emissions, suggests that other mechanisms might be involved. A total origin from Jupiter-Sun orbital interactions would not accommodate periods when there has been flattened solar activity such as during the Maunder Minimum during the years 1645 to 1715 [16].

One possible alternative explanation is that the solar cycle is an intrinsic "standing wave" or resonance that has been induced by the movement of the Solar System at this approximate velocity during its multiple revolutions around the galactic center during the last $\sim 4.5$ billion years. For this condition to have emerged there must be repetitive gradients within the spatial organization. The resulting different quantities of energy from the universal pressure would be encountered in a quasi-systematically manner.

If there were a standing wave that has been initiated by the recursive encounter of an intrinsic gradient in the universal parameter, the first order periodicity could be estimated by the distance of movement of the Sun within 10.4 years $\left(\sim 3.28 \cdot 10^{8} \mathrm{~s}\right)$ at a velocity of $2.42 \cdot 10^{5}$ $\mathrm{m} \cdot \mathrm{s}^{-1}$. This distance is $7.9 \cdot 10^{13} \mathrm{~m}$. According to Gurnett et al [17] interpretation of the observations of the densities of interstellar plasma by Voyager 1, the boundary between solar plasma and much cooler interstellar medium is estimated to be about $1.4 \cdot 10^{13} \mathrm{~m}$.

These observations would be consistent with a heliopause that reflects the distance traversed in an average solar activity cycle through intragalactic space. Leading a classic 
standing wave one would expect "boundary phenomena" that are induced by the persistent encountering of the intrinsic spatial gradient in space, which on average would be $\sim 8 \cdot 10^{13} \mathrm{~m}$. The Oort cloud system between 3 and $7 \cdot 10^{14} \mathrm{~m}$ from the Sun could accommodate this expectation.

However if this spatial gradient was fixed the solar cycle would not range between 7 to 14 years and there would have been no Maunder Minimum with a duration in the order of 75 years. From the context of Borowski's Gravitational Theory [2], the available energy from dark matter would be a function of the vectorial distance from nearest masses. This would change as the Solar System traversed its orbit.

The existence of longer periodicities in the solar cycle has been known for decades. Dewey [18] discussed the $\sim 80$ year cycle that was found by several authors to range between 79 years and 89 years. He concluded that the actual period was more proximal to 88 years. Mikulecky [19], expanding upon A. L. Tchijevsky's coupling of solar activity to human activity, presented evidence of an approximately 500 year periodicity.

\section{INFERENCE OF SECTOR FEATURES}

If the intrinsic periodicity of the solar cycle is coupled to a standing wave associated with the orbital velocity around the galactic center, then inferences about that center's "structure" might be possible. The Sun's distance from the center is about 9,000 Parsecs or $2.77 \cdot 10^{20} \mathrm{~m}$; the orbital distance assuming minimal eccentricity is $1.74 \cdot 10^{21} \mathrm{~m}$. The distance traversed in one solar cycle, assuming an average of 10.4 years is $7.9 \cdot 10^{13} \mathrm{~m}$. This means that the arc is $4.5 \cdot 10^{-8}$ of the total perimeter.

A conservative estimate for the numbers of stars in the galaxy to which the Sun belongs is $\sim 10^{11}$ and assuming each averages the Sun's mass of $\sim 10^{30} \mathrm{~kg}$, the total mass is $\sim 10^{41} \mathrm{~kg}$. The corresponding radius of the singularity according to Schwarzschild:

$$
\mathrm{R}=2 \mathrm{GMc}^{-2} \quad(2)
$$

where $\mathrm{G}$ is the Gravitational Constant, $\mathrm{M}$ is the mass and $\mathrm{c}$ is the velocity of light $\left(3 \cdot 10^{8} \mathrm{~m} \cdot \mathrm{s}^{-}\right.$ ${ }^{1}$ ), is between 1.4 and $1.5 \cdot 10^{14} \mathrm{~m}$ with a perimeter of $\sim 9.3 \cdot 10^{14} \mathrm{~m}$.

Assuming the origin of the spatial gradient in universal pressure and submatter structure is a more or less regular sector expansion, the width of the condition within that singularity that produces the solar cycle periodicity at the Sun's distance would be $\left(4.5 \cdot 10^{-8}\right) \cdot 9.3 \cdot 10^{14} \mathrm{~m}$ or $4.1 \cdot 10^{7} \mathrm{~m}$, or, with the range of the circumference of the earth. The equivalent frequency, assuming c, is about $7.3 \mathrm{~Hz}$ for this value. The possibility that this precision may be spurious because of the estimate for the numbers of stars in the Milky Way is acknowledged.

However the actual duration of solar cycles have ranged from 7 to 17 years which would be (assuming a constant velocity) the equivalent of $5.33 \cdot 10^{13} \mathrm{~m}$ to $1.29 \cdot 10^{14} \mathrm{~m}$ or an arc segment of the perimeter between $3.06 \cdot 10^{-8}$ to $0.74 \cdot 10^{-7}$. The corresponding band along the circumference of the singularity would be between $2.8 \cdot 10^{7} \mathrm{~m}$ to $6.9 \cdot 10^{7} \mathrm{~m}$ with equivalent frequencies between 10.7 and $4.3 \mathrm{~Hz}$. For the "88 year cycle" reported by Dewey [18], the traversed distance would be $6.71 \cdot 10^{14} \mathrm{~m}$ or an arc $3.86 \cdot 10^{-7}$. At the level of the singularity, this would be a distance of $3.6 \cdot 10^{8} \mathrm{~m}$, or a standing wave of about $0.8 \mathrm{~Hz}$.

It may be instructive that a spatial standing wave around the singularity that reflects the hydrogen line frequency $(1.42 \mathrm{GHz})$ would be the expected $2.11 \cdot 10^{-1} \mathrm{~m}$ and would constitute 
$0.23 \cdot 10^{-15}$ of an arc its circumference. At the distance of the Solar System, the equivalent proportion of that arc for the orbit would be $3.95 \cdot 10^{5} \mathrm{~m}$. With a velocity of $2.42 \cdot 10^{5} \mathrm{~m} \cdot \mathrm{s}^{-1}$, the unit time would be between 1 and $2 \mathrm{~s}$. One form of visualization of the space-time structure of the surface area of the event horizon or boundary of the central singularity would be similar to the gyral patterns of the human brain within which local more regular punctate curvatures erupt along sulcal troughs [20].

\section{EVIDENCE OF CONSEQUENCES FROM MOVEMENTS}

The product of velocity $\left(\mathrm{m} \cdot \mathrm{s}^{-1}\right)$ and pressure $\left(\mathrm{kg} \cdot \mathrm{m}^{-1} \cdot \mathrm{s}^{-2}\right)$ is $\mathrm{kg} \cdot \mathrm{s}^{-3}$ which is the equivalent of $\mathrm{W} \cdot \mathrm{m}^{-2}$. The effects upon the earth's upper atmosphere of the Solar System's velocity around the galactic center at $2.42 \cdot 10^{5} \mathrm{~m} \cdot \mathrm{s}^{-1}$ and the universal pressure of $1.5 \cdot 10^{-10}$ $\mathrm{kg} \cdot \mathrm{m}^{-1} \mathrm{~s}^{-2}$ is $3.63 \cdot 10^{-5} \mathrm{~W} \cdot \mathrm{m}^{-2}$ or, as light, the equivalent of about $25 \mathrm{mLux}$. Such an irradiant flux density should be evident.

Even if only $10 \%$ of that irradiance was within the visible wavelengths, the magnitude would be similar to the diffuse light attributed contemporarily to air glow or night glow. There are multiple explanations for the effect which range from luminescence from the impact of cosmic rays to the chemical luminescent reactions between oxygen, nitrogen and hydroxyl groups within the energetic upper atmosphere $(>100 \mathrm{~km}$ altitude). This calculation suggests that a component of this "glow" could originate from the consequences of the earth moving through intragalactic space and the universal pressure.

The second consequence of this movement would be a range of energy that would be effective at quantities sufficient to influence the dynamics that affect fundamental matter.

The product of the earth's mass $\left(\sim 6 \cdot 10^{24} \mathrm{~kg}\right)$ and the square of the velocity $\left(5.85 \cdot 10^{10}\right.$ $\left.\mathrm{m}^{2} \cdot \mathrm{s}^{-2}\right)$ is $3.51 \cdot 10^{35} \mathrm{~J}$. Within the volume of the earth $\left(1.09 \cdot 10^{21} \mathrm{~m}^{3}\right)$ the density would be $3.2 \cdot 10^{14} \mathrm{~J} \cdot \mathrm{m}^{-3}$.

Assuming the universal increment of energy, $\sim 1 \cdot 10^{-20} \mathrm{~J}$, which is the solution of multiplying the average fundamental force within Planck's volume across the neutral hydrogen line [21], the increment of space most affected by this density can be estimated. The quotient of $1 \cdot 10^{-20} \mathrm{~J}$ divided by $3.2 \cdot 10^{14} \mathrm{~J} \cdot \mathrm{m}^{-3}$ is $0.29 \cdot 10^{-34} \mathrm{~m}^{3}$ or a width of about 3.07 picometers which is within the range of the Compton wavelength $(2.4 \mathrm{pm})$ for an electron. In fact if the universal increment were $0.5 \cdot 10^{-20} \mathrm{~J}$, for comparison, the resulting wavelength would be exactly the Compton value.

The equivalent frequency for this wavelength, obtained by dividing this value into $\mathrm{c}$, is $0.98 \cdot 10^{20} \mathrm{~Hz}$ for which the quantum energy equivalent when multiplied by Planck's constant $\left(6.626 \cdot 10^{-34} \mathrm{~J} \cdot \mathrm{s}\right)$ is $6.47 \cdot 10^{-14} \mathrm{~J}$. The mass equivalent is $7.2 \cdot 10^{-31} \mathrm{~kg}$ which is remarkably approximate to the mass of an electron. Obviously, the solution for $0.5 \cdot 10^{-20} \mathrm{~J}, 8.18 \cdot 10^{-14} \mathrm{~J}$, is $9.1 \cdot 10^{-31} \mathrm{~kg}$, the mass of the classic electron. This solution would suggest that the energy available at the lengths and energies in which electrons operate within the earth's mass could be modulated by its velocity as a component of the Solar System as it moves around the galactic center.

Not surprisingly, the energy density from the mass of the sun moving at this velocity $\left(1.17 \cdot 10^{41} \mathrm{~J}\right)$ within that volume $\left(1.41 \cdot 10^{27} \mathrm{~m}^{3}\right)$ produces a similar effect. When this energy density $\left(8.2 \cdot 10^{13} \mathrm{~J} \cdot \mathrm{m}^{-3}\right)$ is divided into the universal increment of energy, the resulting volume is $1.22 \cdot 10^{-34} \mathrm{~m}^{3}$. The resulting linear distance is $\sim 5 \mathrm{pm}$. 


\section{CONCLUSIONS}

The contribution from movement through "different space" as the Solar System traverses its orbit around the galactic center produces an intrinsic energy that can potentially modulate the interaction between the electrons that constitute the mass of the earth and the Sun. The passive movement of the earth through the universal pressure is sufficient to accommodate the energy released within the crust by seismic events and to produce gradual increases (or decreases) in ocean temperatures that are congruent with current observations. The available energy also matches recent increases in the amplitude of geomagnetic activity. There is very likely to be a variable intensity of the universal pressure that produces quasiregularity in the spatial periodicity of these values that strongly influences the duration of solar cycles (and their absence) as well as variations in global temperature and earthquakes. The periodicity of this complexity of sub-matter space through which the Solar System moves might be determined by the extended structure of the boundary of event horizon of the galaxy's singularity.

\section{Acknowledgments}

Thanks to Professor David Vares for supplying the data from U.S.G.S. files.

\section{References}

[1] S. P. Wyatt, Principles of Astronomy, Allyn and Bacon, Boston, 1964.

[2] M. A. Persinger, International Letters of Chemistry, Physics and Astronomy 11 (2014) $18-23$.

[3] T. Borowski, International Letters of Chemistry, Physics and Astronomy 11 (2013) 44-53.

[4] M. A. Persinger, International Journal of Astronomy and Astrophysics 2 (2012) 125-128.

[5] M.A. Persinger, Journal of Physics, Astrophysics and Physical Cosmology 3 (2009) 1-3.

[6] D. A. E. Vares, M. A. Persinger, International Journal of Geosciences 4 (2013) 1321-1325.

[7] H. E. Puthoff, Physical Review A 39 (1989) 2333-2342.

[8] M. Bordag, U. Mohideen, V. M. Mostepanenko, Physics Reports 353 (2001) 1-205.

[9] M. A. El-Borie, S. S. Al-Thoyaib, International Journal of Physical Sciences 1 (2006) 67-74.

[10] K. Lassen, E. Christensen, Journal of Atmospheric and Terrestrial Physics 57 (1995) 835-839.

[11] M. A. Persinger, International Journal of Physical Sciences 4 (2009) 44-46.

[12] M. Lockwood, R. Stamper, M. D. Wild, Nature 399 (1999) 437-439.

[13] L. K. Fenton, P. E.Geissler, R. M. Haberie, Nature 446 (2007) 646-649.

[14] G. Woodbury, Physical Chemistry Brooks/Cole, Pacific Grove, 1997.

[15] F. M. White, Heat and Mass Transfer Addison-Wesley, Reading (Mass), 1991. 
[16] I. Nicholson, The Sun, Rand McNally, Chicago, 1982.

[17] D. A. Gurnett, W. S. Kurth, L. F. Bulaga, N. F. Ness, Science 341 (2013) 1489-1492.

[18] E. R. Dewey, Cycles, Foundation for the Study of Cycles, Inc., Pittsburg (Penn), 1970.

[19] M. Mikulecky, Neuroendocrine Letters 28 (2007) 749-756.

[20] D. C. Van Essen, H. A. Drury, Journal of Neuroscience, 17 (1997) 7079-7102.

[21] M. A. Persinger, S. A. Koren, G. F. Lafreniere, NeuroQuantology 6 (2008) 262-271. 